\title{
Swing Coupling between Stellar Pulsations and Torsional Alfvén Oscillations
}

\section{T. Zaqarashvili}

School of Mathematics and Statistics, University of St. Andrews, St. Andrews, Fife KY16 9SS, Scotland

Abastumani Astrophysical Observatory, Al. Kazbegi ave. 2a, 380060

Tbilisi, Georgia

\section{B. Roberts}

School of Mathematics and Statistics, University of St. Andrews, St. Andrews, Fife KY16 9SS, Scotland

\begin{abstract}
We suggest a mechanism of energy transmission from stellar radial pulsations into torsional oscillations in a dipole-like magnetic field. We show that the stellar radial pulsations cause a periodical variation of the Alfén speed which leads to the Mathieu equation for torsional Alfén waves. Harmonics with half the frequency of the pulsations have exponentially growing amplitudes. Then the energy supporting the various type of pulsations can be transformed into purely electromagnetic oscillations of the star.
\end{abstract}

\section{Introduction}

Pulsations are found at any stage of stellar evolution along the HertzsprungRussell diagram. They are observed with a range of periods from seconds to years. There can be a number of energy sources supporting the pulsations: radiation, nuclear reactions, convection, etc. The whole of this wide spectrum of stellar pulsations is well studied, both observationally and theoretically (e.g., Gautschy \& Saio, 1995). We study a swing coupling between stellar pulsations and torsional Alfvén oscillations in dipole-like magnetic field, based on the recently proposed swing interaction between longitudinal and transverse waves in a medium (Zaqarashvili, 2001): a periodical variation of the medium parameters due to the longitudinal waves affects the phase velocity of transverse waves and causes the resonant energy transformation into certain harmonics.

\section{Swing coupling between pulsations and torsional oscillations}

We use a Cartesian frame, where the $x$ axis has the toroidal direction, the $y$ axis is directed from the equator to the pole and the $z$ axis is directed perpendicular to the stellar surface. In this frame the poloidal magnetic field has only the $y$ component, $B_{y}$, at the equator. Then the linear radial velocity field of the 
pulsating star at the equator may be written as:

$$
u_{z}=\alpha F(z) \sin \left(\omega_{0} t\right),
$$

where $\alpha$ is the amplitude, $\omega_{0}$ is the eigenfrequency and $F(z)$ governs the eigenfunctions of the pulsation.

Corresponding density and magnetic field perturbations are:

$$
\begin{gathered}
\delta \rho=\frac{\alpha}{\omega_{0}} \cos \left(\omega_{0} t\right) F_{\rho}(z), \quad b_{y}=\frac{\alpha}{\omega_{0}} \cos \left(\omega_{0} t\right) F_{b}(z), \\
F_{\rho}(z)=\frac{\partial}{\partial z}\left(\rho_{0}(z) F(z)\right), \quad F_{b}(z)=\frac{\partial}{\partial z}\left(B_{y}(z) F(z)\right) .
\end{gathered}
$$

Then the modified equations of the Alfvén waves lead to the Mathieu equation (the terms of order $\alpha^{2}$ are neglected) for the toroidal component of the magnetic field perturbation

$$
\frac{\partial^{2} h_{x}}{\partial t^{2}}+\left[\frac{B_{y}^{2} k_{y}^{2}}{4 \pi \rho_{0}}+\alpha \Psi(z) \cos \left(\omega_{0} t\right)\right] h_{x}=0,
$$

where $k_{y}$ is the wave number of torsional Alfén waves and

$$
\Psi(z)=\frac{B_{y}^{2} k_{y}^{2}}{4 \pi \rho_{0} \omega_{0}}\left[2 \frac{F_{b}}{B_{y}}-\frac{F_{\rho}}{\rho_{0}}\right]-\frac{\omega_{0}}{2} \frac{F(z)}{B_{y}} \frac{\partial B_{y}}{\partial z} .
$$

The equation has an exponentially growing solution if

$$
\omega_{A}=\frac{B_{y} k_{y}}{\sqrt{4 \pi \rho_{0}}}=\frac{\omega_{0}}{2} .
$$

\section{Conclusions}

The amplification of torsional oscillations in a dipole-like magnetic field due to pulsations opens a new area for investigation of stellar interiors. It means that a non-electromagnetic energy supporting any type of pulsation can be transmitted into purely magnetic oscillations with the half-frequency of pulsations.

The swing coupling between pulsations and torsional oscillations may lead to variations in the Zeeman splitting for early type stars (without convection zones), while for later type stars (with convection zones) it may lead to a variation in chromospheric activity. Both of these effects can be probed by observations.

Acknowledgments. The work partially was supported by the Royal Society/NATO Postdoctoral Fellowship programme and INTAS grant 97-31931.

\section{References}

Gautschy, A. \& Saio, H. 1995, ARA\&A, 33, 75

Zaqarashvili, T.V. 2001, ApJ, 552, L81 